\section{Future of Personalised Medicine: Pharmacoprinting of the Polypill}

Sir,

Multiple medications are required to treat ailments like cancer and cardiovascular diseases. This leads to the hassle of remembering to take multiple drugs at various times and; hence, patient compliance is affected. A possible alternative is the formation of a "polypill", which is the combination of various drugs, in one single capsule. A polypill is a medication that is a drug product in pill form (i.e., tablet or capsule) that combines multiple active pharmaceutical ingredients. It is generally manufactured as a fixed-dose combination (FDC) product for the treatment or prevention of chronic illness. ${ }^{1}$

The notion of the formation of a polypill, using the technique of three-dimensional (3D) printing, has been successfully explored. Khaled et al. have successfully utilised 3D printing for the formation of a tailor-made drug, using captopril, nifedipine and glipizide formulation, to treat patients with diabetes and hypertension. ${ }^{2}$

The 3D printing is the production of a layer by layer formulation of $3 \mathrm{D}$ products based on a digital design. The conception of 3D printing, also referred to as rapid prototyping (RP), solid-freeform technology (SFF), or additive manufacturing (AM), has been a groundbreaking phenomenon. Charles Hull developed the earliest technique "stereolithography" in the early 1980 s. Multiple 3D printing methods are gaining popularity, specifically Inkjet printing, selective laser sintering (SLS), fused deposition modelling (FDM), and laminated object manufacturing (LOM). ${ }^{3}$

Many elderly patients are confused and take their pills haphazardly and/or in the wrong dosage. A polypill would be expected to help this group of patients considerably. Printing specialised tablets for patients, with certain specific allergies might also be possible. A visual/tactile identifier can also be incorporated into the 3D printed tablets to aid sight-compromised patients. Hence, different individual requirements of patients can be met and this allows a more personalised approach to pharmacotherapy. 4

With the advent of 3D printing of polypills, medicine will become more adapted for every individual and bring about a greater degree of patient compliance, as every patients' need would be addressed with their specific drug formulation, which would make them feel included, bringing a whole new dimension to the future of personalised medicine. These 3D printers can be kept in the physician's office, the pharmacy or even at home.
Having printers at these facilities will allow patients to be more involved in the process of drug making. But, it does have a fair share of ethical and possible technical issues. The decision about who should be allowed to print their own drugs will be a moral dilemma. Some people might abuse pharmacoprinting and use it for mass production for selling purposes, while others may print the drugs the wrong way and cause adverse drug reactions. Moreover, 3D printed tablets look and feel vastly different from normal tablets, and this might affect the patient's trust in pharmacoprinting. Personalised polypills would increase the patient's role in drug making and would ultimately shift the patient-physicianpharmacist dynamics, which may become overwhelming in the long term. 5

Furthermore, the subsequent costs of a polypill are likely to be much greater than the generic components. Costeffective-ness is likely to decrease as the number of components increases. A polypill also has potential for harmful drug interactions, as some drugs are better tolerated alone and not in combination; hence, more research would be required before these formulations are introduced to the general public. Extensive reeducation of primary care and specialist physicians would be required so that they actively participate in the formation of tailor-made combinations for their individual patients, which would also be costly. 1

Hence, the idea of a polypill and the ease of its production with the arrival of a 3D printer is a very lucrative venture, and if carefully researched, can yield rewarding results. Though, there are drawbacks due to use of financial resources and possible drug interactions, but if the problems are properly addressed, these could lead to a forthcoming change in the field of personalised medicine and pharmacotherapy. Some possible interventions can be that there be a thorough process for the selection of patients who are allowed 3D printers at home. Raw materials used for the medicinal drugs should be rationed monthly, so that people have enough and not more for the drugs they need. A self-regulating setting can be installed, where only the specific amount of drug is made per time, this will prevent mass production. An alarm can be incorporated into the 3D printers, which may warn the consumer of a possible adverse drug interaction. Physicians and pharmacists should have a more active role in the process and have annual surveillance of the $3 \mathrm{D}$ printers and the materials that are printed. More research should be done for different combinations before they are incorporated into a single polypill. Awareness is needed for the responsible use of pharmacoprinting so that this technique is not abused, and truly consumed to its maximum potential. Investment in this endeavour would be productive as it will set the stage for the rising field of individualistic medicine. 


\section{CONFLICT OF INTEREST:}

Authors declared no conflict of interest.

AUTHORS' CONTRIBUTION:

$\mathrm{SH}$ : Wrote the article and performed extensive literature review.

SMT: Suggested the topic of discussion, revised the article before submission.

$\mathrm{SH}$ : Reviewed the article before final submission, arranged and formatted the layout.

\section{REFERENCES}

1. Sleight $P$, Pouleur $H$, Zannad F. Benefits, challenges, and registerability of the polypill. Eur Heart $J$ 2006; 27:1651-6.

2. Khaled SA, Burley JC, Alexander MR, Yang J, Roberts CJ. 3D printing of tablets containing multiple drugs with defined release profiles. Int J Pharm 2015; 494:643-50.

3. Gross BC, Erkal JL, Lockwood SY, Chen C, Spence DM. Evaluation of $3 \mathrm{D}$ printing and its potential impact on biotechnology and the chemical sciences. Anal Chem 2014; 86: 3240-53.
4. Khaled SA, Burley JC, Alexander MR, Yang J, Roberts CJ. 3D printing of five-in-one dose combination polypill with defined immediate and sustained release profiles. J Control Release 2015; 217:308-14.

5. Kaae S, Lind JL, Genina N, Sporrong SK. Unintended consequences for patients of future personalized pharmacoprinting. Int J Clin Pharm 2018; 40:321-4.

Sana Habib ${ }^{1}$, Syed Maaz Tariq ${ }^{2}$ and Sara Habib ${ }^{3}$

1 Medical Student, Dow University of Health Sciences, Karachi, Pakistan

2 Medical Student, Jinnah Sindh Medical University, Karachi, Pakistan

3 Department of Neurosurgery and Neurocritical Care, Thomas Jefferson University, Suite 332, PA, USA

Correspondence to: Dr. Sara Habib, Department of Neurosurgery and Neurocritical Care, Thomas Jefferson University, 901 Walnut Street, Suite 332, PA, USA

E-mail: sara.habib@jefferson.edu

Received: January 22, 2019; Revised: March 06, 2019; Accepted: March 28, 2019 